\title{
Dissipative soliton resonance as a guideline for high-energy pulse laser oscillators
}

\author{
Philippe Grelu, ${ }^{1, *}$ Wonkeun Chang, ${ }^{2,3}$ Adrian Ankiewicz, ${ }^{2}$ Jose M. Soto-Crespo, ${ }^{4}$ and Nail Akhmediev ${ }^{2}$ \\ ${ }^{1}$ Laboratoire Interdisciplinaire Carnot de Bourgogne, UMR 5209 CNRS, Université de Bourgogne, \\ 9 Avenue A. Savary, BP 47870, Dijon CEDEX 21078, France \\ ${ }^{2}$ Optical Sciences Group, Research School of Physics and Engineering, Institute of Advanced Studies, \\ The Australian National University, Canberra, Australian Capital Territory 0200, Australia \\ ${ }^{3}$ Present address: Max-Planck Institute for the Science of Light, Guenther-Scharowsky Str. 1/24, \\ D-91058 Erlangen, Germany \\ ${ }^{4}$ Instituto de Óptica, C.S.I.C., Serrano 121, 28006 Madrid, Spain \\ *Corresponding author: philippe.grelu@u-bourgogne.fr
}

Received June 8, 2010; revised August 12, 2010; accepted September 9, 2010;

posted September 16, 2010 (Doc. ID 129813); published October 18, 2010

\begin{abstract}
Dissipative soliton resonance (DSR) occurs in the close vicinity of a hypersurface in the space of parameters of the equation governing propagation in a dissipative nonlinear medium. Pulsed solutions can acquire virtually unlimited energies as soon as the equation parameters converge toward that specific hypersurface. Here we extend previous studies that have recently unveiled DSRs from the complex cubic-quintic Ginzburg-Landau equation. We clearly confirm the existence of DSR for a wide range of parameters in both regimes of chromatic dispersion, and we establish general features of the ultra-high-energy pulses that can be found close to a DSR. Application to high-energy mode-locked fiber oscillators is discussed. (C) 2010 Optical Society of America OCIS codes: $190.5530,140.4050$.
\end{abstract}

\section{INTRODUCTION}

For the past few years, there has been an intense experimental research activity in developing high-energy single-pulse oscillators within passively mode-locked fiber laser technology. The energy available in short or ultrashort coherent pulses has increased by several orders of magnitude in less than a decade, from the nanojoule to the microjoule level [1-6]. In the course of scaling up the output power available from compact fiber laser oscillators, several key strategies have been identified. In addition to the improvement of high-power pumping laser diodes and double-clad rare-earth-doped fiber amplifiers [7], specific strategies are certainly required to avoid the single-pulse energy limitations imposed by pulse breakup and multiple pulsing that result from an excess of nonlinear phase shift per round trip.

To reduce nonlinearity impairments, the use of largemode-area photonic crystal fibers has become widespread $[6,8]$. In addition, the available energy from the pumping source can be converted into higher-energy pulses when the cavity fundamental repetition rate is decreased. In that respect, the prospect of developing ultralong passively mode-locked fiber lasers is particularly attractive [4], as long as the stability issue of kilometer-long cavities can be resolved satisfactorily. But the point is that most high-energy-pulse strategies consist of favoring the circulation of highly chirped pulses in normally dispersive fiber links $[3,9,10]$. Cavity designs and corresponding experiments have successively considered dispersionmanaged laser cavities [11], and wave-breakup-free, selfsimilar, and all-normal-dispersion fiber lasers $[3,12,13]$. Although, in essence, the common trend corresponds to incorporating the well-established concept of chirpedpulsed amplification inside the laser oscillator $[9,14,15]$ the solution is not so obvious since, unlike the use of an external amplifier, the input field at the amplifier section is not known in advance-it results from a global dynamical balance of the various physical effects at play inside the whole laser cavity. A model of the laser cavity will generally correspond to a rather extended set of parameters; and, of course, several classes of cavity designs can be considered.

Hence, it is useful to map the possible dynamics that can be found out of simple master equations for modelocked lasers. Due to its applicability to various modelocked lasers schemes and its universal features that allow correspondence in several fields of nonlinear science, the complex cubic-quintic Ginzburg-Landau equation (CGLE) is a master equation of choice. Each of its terms describes, in a distributed way, a given physical effect at play in a mode-locked laser [16,17].

The study of pulsed dynamics from the CGLE has allowed a natural explanation of several unusual singleand multiple-pulse dynamics that have been observed in mode-locked lasers, such as self-pulsations [18], explosions [19], and the formation of multi-soliton complexes [20,21]. Investigations based on the CGLE have been crucial in the development of the concept of dissipative solitons [17], which is now a well-established notion in the community of physicists. It provides, for instance, a clear frame for understanding the existence of stable ultrashort laser pulses produced in normally dispersive mode-locked laser cavities.

During numerical explorations of dissipative soliton so- 
lutions in the parameter space of the CGLE, a certain region of the equation parameters was found where the energy of single pulses increased indefinitely whenever the equation parameter converged to this region [10]. A set of parameters where the soliton energy diverges to infinity was called a dissipative soliton resonance (DSR) [22]. Found in the normal dispersion regime, it initially required a positive quintic reactive nonlinearity to appear $[10,22]$, but then DSR was also revealed in the chromaticdispersion-free $(D=0)$ regime, along with negative quintic reactive nonlinearity [23]. The existence of DSR with negative quintic reactive nonlinearity, which corresponds to the higher-order CGLE, seems indeed more physical, although we shall discuss further the possible relevance of a DSR to the positive quintic reactive nonlinearity. In addition, convincing arguments were advanced in [23] for the existence of DSR in the anomalous dispersion regime, although the existence of DSR with markedly anomalous dispersion was not obviously shown. Recently there has been experimental support for this idea, with the demonstration of pulses that are approximate examples of DSR [24].

Finding the region of parameters where DSR exists is a tedious task, as it requires an enormous number of numerical simulations. Although this work can be simplified with approximations of the pulse shape, finding the set of parameters which provides for the simultaneous existence of the effect in the normal and anomalous regimes still needs numerical simulations.

In this work we find, numerically, such a large DSR region, and we detail the pulse shapes that may appear if this range of parameters is taken. We then present the general pulse features in the vicinity of a DSR that are common to both dispersion regimes and to all numerical observations of DSR reported so far. We show that an elaborate trial function allows for an excellent retrieval of the DSR location in the anomalous dispersion part of the explored parameter domain. Finally, we discuss the impact of our work in the context of related ongoing theoretical and experimental works.

\section{RESONANCES IN ANOMALOUS AND NORMAL DISPERSION REGIMES}

We have extended previous numerical studies in the parameter space of the CGLE, in order to clearly demonstrate the existence of DSRs for both dispersion signs over a wide range of parameters, especially in the anomalous dispersion domain. For the sake of clarity, let us recall the distributed cubic-quintic Ginzburg-Landau equation under study:

$$
i \psi_{z}+\frac{D}{2} \psi_{t t}+|\psi|^{2} \psi+\nu|\psi|^{4} \psi=i \delta \psi+i \epsilon|\psi|^{2} \psi+i \beta \psi_{t t}+i \mu|\psi|^{4} \psi
$$

The optical envelope $\psi$ is a complex function of two real variables, i.e., $\psi=\psi(t, z)$, where $t$ is the retarded time in the frame moving with the pulse, and $z$ is the propagation distance. The left-hand-side of Eq. (1) contains the conservative terms, viz., $D>0(D<0)$ stands for the amount of anomalous (normal) dispersion, and $\nu$ represents the quintic reactive Kerr nonlinearity. The right-hand-side includes all dissipative terms: $\delta, \epsilon, \beta$, and $\mu$ are the coefficients for linear loss (if negative), nonlinear gain (if positive), spectral filtering, and saturation of the nonlinear gain (if negative), respectively.

A multiplicity of numerical trials leads us to the following optimal set of four parameters: $\delta=-0.05, \beta=0.4$, $\nu=-0.08, \mu=-0.05$. The signs for $\delta, \beta$, and $\mu$ can be taken only the way they are here, as explained in our previous works. However, the sign for $\nu$ still admits a certain freedom. The reactive quintic nonlinearity $\nu$ was chosen to be negative in accordance with the result of a previous study, i.e., Eq. (14) in [22]. Indeed, the reduced system constructed using the chirped super-Gaussian type function indicated that $\nu$ should be negative to shift the DSR into the anomalous dispersion regime.

We varied the remaining two parameters and studied the energy of stable pulse solutions in the twodimensional parameter space $(D, \epsilon)$. The results of this numerical exploration are shown in Fig. 1, which displays in color scale (online) or gray scale (in print) the region of existence of solitons and the energy of stable solitons in this region.

The DSR curve in this plot is the upper boundary of the soliton existence region. It is easy to see that the DSR curve runs continuously from normal to anomalous dispersion and occupies significant intervals in each of these regions. This is considerably different from the results found in $[10,22]$.

Each point of this upper boundary corresponds to a limit where the energy of dissipative solitons tends to become infinite. This may be seen if we plot the pulse energy versus either of the variables $\epsilon$ and $D$. In particular, we can fix the value of $\epsilon$ and plot $Q$ versus $D$. We can also observe the pulse shape variations when $D$ changes along the chosen line on the $\epsilon-D$ plane. Two of these lines are marked in Fig. 1 by stars and vertical crosses. One of the two sets is chosen to illustrate the pulse transformations when approaching the resonance line in the normal dispersion regime, while the other set is chosen in the anomalous dispersion regime.

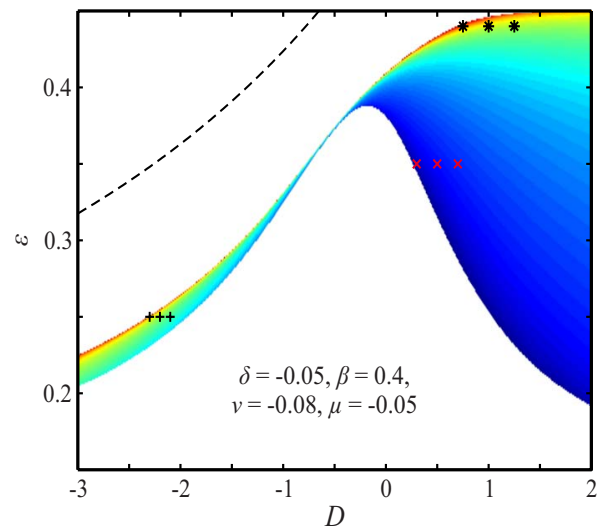

Fig. 1. (Color online) Contour plot of the energy of stable soliton solution in the two-dimensional parameter space of (dispersion $D$, nonlinear gain $\epsilon$ ) in the CGLE. Color scales the pulse energy from low (blue) to high (red) levels. Pulse solutions for the three sets of parameter values denoted by vertical crosses "+," stars "*," and oblique crosses " $\times$ " are detailed in the following figures. The dashed line is an analytical approximation to the resonance curve [22]. 
In the normal dispersion regime, the nonlinear gain parameter is chosen to be $\epsilon=0.25$, while the dispersion parameter takes the following three values: $D=-2.1,-2.2$, and -2.3 . The curves representing the corresponding pulses are shown in Fig. 2. We can see that when changing $D$ from -2.1 to -2.3 , the pulse duration increases by a factor of 4 , whereas the increase in pulse amplitude is limited [see Fig. 2(a)]. At the same time, the spectral width hardly changes [see Fig. 2(c)].

This type of pulse transformation is quite general near any other point of the DSR curve. Indeed, the increase in pulse width entails an increase in pulse energy without meaning a trivial transition from pulse solutions to continuous waves (cw's). Also, the frequency chirp is another important feature of the pulse. Moving a little further in the parameter space to the location of the resonance $(D$ $\simeq-2.32$ ) entails an abrupt increase in the soliton pulse width and energy. Chirped pulses of virtually unlimited energy can thus be found in the CGLE model in the region with normal dispersion.

When shifting $\epsilon$ above the value of 0.41 , DSR occurs in the anomalous dispersion regime. We illustrate the existence of DSR in the anomalous dispersion regime by choosing $\epsilon \approx 0.44$, which provides a DSR when $D \simeq 0.74$. Pulse shape, chirp, and the spectral curves are shown in Fig. 3 for the following three dispersion values: $D=1.25$, 1.00 , and 0.75 .

In these examples, the flat-top effect for pulses close to a DSR appears to be more pronounced than for the normal dispersion case [see Fig. 3(a)]. The chirp is linear across the flat-top part of the pulse. This corresponds to a parabolic phase profile-a useful feature that allows, in principle, efficient pulse compression through common dispersive components such as grating pairs. As in the normal dispersion regime, the increase in pulse duration
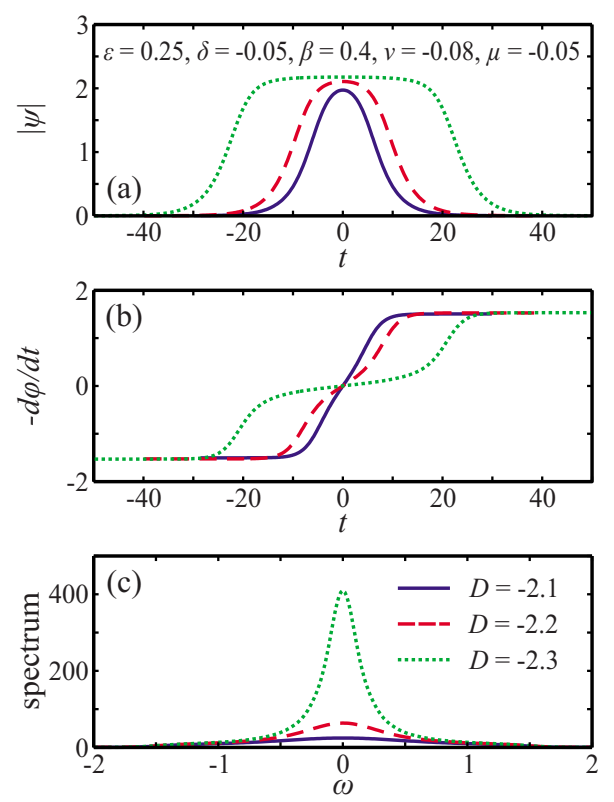

Fig. 2. (Color online) (a) Pulse shape, (b) chirp, and (c) the spectrum transformations near the DSR in the normal dispersion regime. The points on the $\epsilon-D$ plane which correspond to these curves are shown by the vertical crosses (+) in Fig. 1.
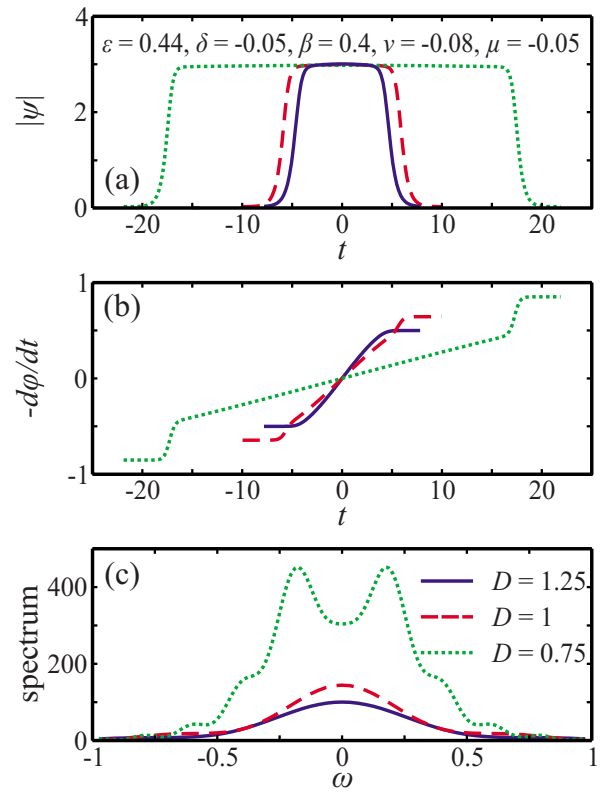

Fig. 3. (Color online) (a) Pulse shape, (b) chirp, and (c) the spectrum transformations near the DSR in the anomalous dispersion regime. The points on the $\epsilon-D$ plane which correspond to these curves are shown by the stars (*) in Fig. 1 .

when approaching the DSR curve is not accompanied by a reduction in the spectral width. This means that the changes in pulse profile do not correspond to the trivial transition toward a cw solution.

When changing $D$ from 1.25 to 0.75 , the optical spectrum broadens and becomes more structured [see Fig. 3(c)]. This is partly related to the evolution of the phase profile across the pulse that comes along with the flat-top effect as can be seen from Fig. 3(b). At the same time, the frequency chirp is almost linear along the flat-top part of the pulse. It undergoes abrupt changes only at the two pulse ends.

Let us confirm that the chirp sign in the central part of the pulses is the same across the entire region of stable solitons in all dispersion regimes as can be seen from the plots in Figs. 2(b), 3(b), and 4(b): the pulses are redshifted to the leading edge. Although this fact seems counterintuitive in the anomalous domain, it is required by the continuity of the DSR curve which goes from the normal to the anomalous dispersion domain.

The lower boundary of the dissipative soliton existence region is the curve where solitons lose their stability. The solitons at this edge become weaker and do not have sufficient pump energy to support them. The pulse changes close to this boundary are illustrated in Fig. 4. Parameters $\epsilon$ and $D$ are chosen at the points shown by oblique crosses " $X$ " in Fig. 1. Specifically, $\epsilon=0.35$ and $D=0.7,0.5$, and 0.3 . The boundary itself is located at $D \simeq 0.25$. The soliton energy progressively decreases when moving toward the boundary. No stable soliton solutions can be found outside the colored region in Fig. 1. In the interval $-1.5<D<0$, the domain of soliton existence in the parameter space is relatively narrow. It is much wider at other values of $D$, both in the normal and anomalous dispersion regimes. 

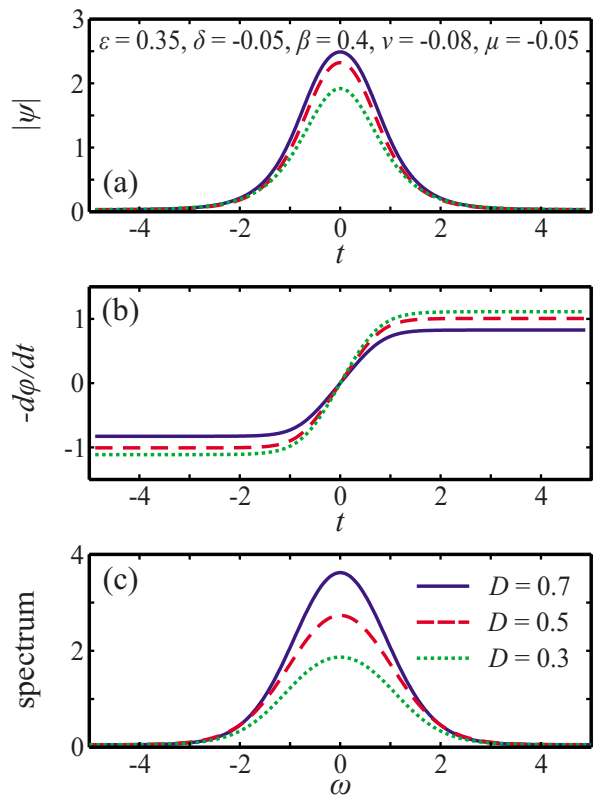

Fig. 4. (Color online) (a) Pulse shape, (b) chirp, and (c) the spectrum transformations near the lower boundary of the region of soliton existence. The points on the $\epsilon-D$ plane which correspond to these curves are shown by the oblique crosses $(\times)$ in Fig. 1 . When moving toward the lower boundary (when $D$ changes from 0.7 to 0.3 ), the soliton energy decreases.

\section{GENERAL DSR FEATURES}

Despite a few differences in the pulse profiles near the DSR curve in the normal and anomalous dispersion regimes, we can point out their common features as well. These are quite general, and they are fully compatible with all previous results $[10,22,23]$. By our definition, a DSR occurs at the set of parameters where the pulse solutions can acquire virtually unlimited energy.

Clearly, using the CGLE model (1), one has to be careful when extrapolating the results to real systems such as mode-locked lasers. We have to remember that there is no global saturation, and that cw solutions with nonzero amplitude also possess an infinite energy. Thus, we need certain criteria in order to distinguish a DSR from a trivial transition from soliton to $\mathrm{cw}$ solutions. Indeed, in all the examples shown above, pulse duration is seen to increase dramatically in the vicinity of a DSR, whereas the pulse amplitude seems to converge to a given plateau value in the pulse flattening process when parameters approach the DSR resonance curve.

Obviously, the pulse spectral width should remain above a significant nonzero lower limit, while the pulse duration goes to infinity. This has several consequences. The pulses should always have frequency chirping. We assume that the spectral width remains finite, which is a reasonable assumption, taking into account both the expected influence of the spectral filtering term with coefficient $\beta$ and the results of existing simulations.

A long pulse plateau is also compatible with quasilinear frequency chirping. Hence, in the limiting case, the amount of chirp should be inversely proportional to the pulse duration. Since the amount of chirp tends to zero when the pulse duration tends to infinity, the influence of the temporal derivatives in the CGLE also tends to zero in the main part of the pulse. As a consequence, the value of the pulse amplitude can be given accurately by solving the CGLE for the cw solution, which becomes a polynomial equation. Nevertheless, we stress the fact that the limiting case is not the $\mathrm{cw}$ solution due to the finite spectral width of the pulse.

More precisely, the pulse energy, $Q=\int_{-\infty}^{\infty}|\psi|^{2} d t$, increases:

$$
Q \rightarrow+\infty
$$

the peak power $P$ reaches a constant value:

$$
P=|\psi|_{\max }^{2} \rightarrow P_{c w}=\frac{\epsilon+\sqrt{\epsilon^{2}-4 \mu \delta}}{-2 \mu},
$$

the pulse length

$$
T \rightarrow+\infty,
$$

while the product of chirp $C=\left(\partial^{2} \phi / \partial t^{2}\right)_{t=0}$ and the pulse length tends to a constant:

$$
C T \rightarrow K(\epsilon, \delta, \mu, \nu, D / \beta)
$$

Relations (2)-(5) define general features of DSRs in the CGLE model. Naturally, relation (2) and the peak power given by Eq. (3) imply relation (4) that is explicitly provided for clarity.

\section{RESONANCE CURVES}

Numerical simulations require special techniques near the DSR, thus making them especially tedious. Finding the exact DSR curves, such as in Fig. 1, for each set of parameters is a lengthy procedure that requires many days of calculations. Using approximations that involve trial functions makes the work much quicker, although the results need to be always checked by numerical simulations of the original equation. Implementing this technique was initiated in [22]. Namely, using the method of moments and a trial function in the form of a chirped superGaussian, an approximate resonance curve was indeed found. The resonance curve in this technique can be written as an explicit expression involving the CGLE parameters. The results provided by this expression are shown in Fig. 1 by a dotted line. It can be seen that the qualitative features of the DSR curve are indeed the same as in numerical simulations. It is hard to expect a better fit, taking into account the rough approximation of the above trial function. However, it is clear that the chirped superGaussian fails to approximate the DSR in the anomalous dispersion part of the domain under study.

In the present work, we made efforts in improving the approximation using a more elaborate trial function developed in [25]. Namely, we have used the following trial function:

$$
\psi(t, z)=A(t) e^{i \varphi(t)+i \Omega z}
$$

with the amplitude function 


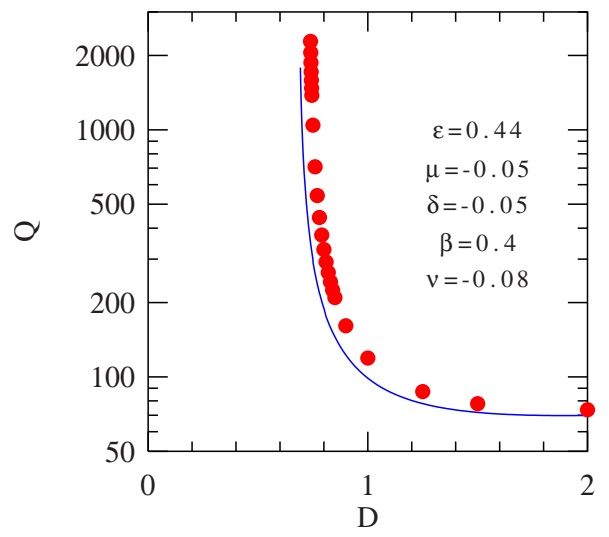

Fig. 5. (Color online) Comparison between numerical results and trial function approximation of the DSR phenomenon. Pulse energy $Q$ is plotted versus dispersion $D$ showing resonance at $D \simeq 0.7$. The blue line is the horizontal cut of the $Q(\epsilon, D)$ surface at $\epsilon=0.44$ in Fig. 1. Red circles are obtained from the approximation that uses the trial function (6).

$$
A(t)=\frac{\sqrt{P}}{\left[1+(1-\eta) \sinh ^{2}\left(\frac{t}{T}\right)\right]^{1 / 2}}
$$

and the phase given by

$$
\varphi(t)=d \ln A(t)+C \int^{t} t^{\prime} A^{2}\left(t^{\prime}\right) d t^{\prime},
$$

where $d$ and $C$ are chirp parameters, and $\eta$ is the shape factor. Clearly, the amplitude function (7) is a good approximation for the pulse shapes having almost rectangular profile when the shape factor tends to 1 . However, a major improvement in DSR approximation comes from the second term in Eq. (8) which provides a linear chirp in the extended part of the pulses near the DSR.

The slice of the surface $Q(\epsilon, D)$ at the line $\epsilon=0.44$ in Fig. 1 is shown in Fig. 5 by the blue line. It is obtained from direct numerical simulations of the CGLE. Red dots represent calculations with the trial function (6) following the same technique as in [25]. It can be seen that function (6) provides a considerably better fit than the superGaussian function. Indeed, the difference in $D$ between the numerical curve and the approximated one is here less than 0.1, while the same difference between the dotted line and exact resonance in Fig. 1 is around 1.5. However, this depends on the particular choice of CGLE parameters. We should note that there is no universal trial function with a limited number of parameters that can be accurate for any arbitrary set of parameters.

\section{DISCUSSIONS AND CONCLUSION}

Conditions (2)-(5) for solitons near the DSR curve describe ultralong high-energy pulses that feature frequency chirping, and that fall inside a given optical amplifier bandwidth. As such, they represent a guideline to increase the pulse energy in an almost unlimited way in a given laser configuration where just a limited number of parameters require fine tuning. Using such fine tuning of cavity parameters to approach the dissipative soliton resonance (DSR), the practical limitation is the available pumping power and conditions for channeling that power into a single pulse. Although we cannot rule out the possibility that higher-order effects could add more practical limitations, the guidelines presented by Eqs. (2)-(5) seem to be robust with respect to most of them. Indeed, the increase in pulse energy does not involve either an increase in the pulse amplitude or an increase in the pulse spectral width. The existence of DSRs found from the study of the general CGLE model reveals that there should be a selfcontained universal route to implement the chirpedpulsed amplification concept inside the laser oscillator itself, namely, scalable high-energy chirped-pulsed oscillators. The goal of developing the principle of these energy-scalable chirped-pulse oscillators has been recently investigated in [15]. Under the main assumption that dispersive effects would dominate dissipative ones, the authors found chirped solitary pulses that are approximate analytical solutions of the CGLE and obey energy scalability in the normal dispersion regime [26]. They also provided convincing comparisons with experiments. Let us remark that the concept of DSR turns out to be more general since it is not limited by the assumption of relatively small dissipative effects, nor it does require positive dispersion to manifest itself.

From the practical point of view, the present work should provide incentives to the design of novel fiber laser cavities that are able to support DSRs. As an original and less expected result, we point out the possibility of operating a mode-locked laser that generates high-energy single pulses in the anomalous dispersion regime as well, provided that the set of other physical effects at play, particularly dissipative ones, is found adequately. This means, in practice, that the saturable absorber should correspond to a specific nonlinear transfer function, although the ways of implementing this transfer function into a cavity could be a difficult task.

When making a practical application of the results obtained from the study of a general distributed master equation such as the CGLE, several important issues require attention. First of all, a real laser system is made of several parts, in which propagation conditions differ. In this respect, it is worthwhile to mention that the DSR does also exist in laser models with parameter management [27]. DSR appears as a robust phenomenon that survives the presence of periodic propagation discontinuities, in general, and dispersion management, in particular. The latter is a commonly used design in mode-locked fiber laser systems.

Second, establishing a relationship between the CGLE parameters and those of the various fiber laser elements is a highly non-trivial task that has been undertaken in a limited number of publications. In [28], the authors used a multi-scale iterative approach to establish the link between the physical parameters of a fiber ring laser mode, locked through nonlinear polarization evolution. This involved the orientation angles of bulk phase plates and the coefficients of the CGLE equation. In particular, calculations included the quintic dissipative term $\mu$, but no correspondence was found for the quintic reactive nonlinearity $\nu$. This work was recently extended in [29] where the authors found relations for both $\mu$ and $\nu$ quintic terms. In 
this work, the nonzero effective quintic reactive nonlinearity appears from the iterative averaged approach, whereas no saturation of the Kerr effect is expected in the optical fibers used in practice. This work provides an additional support to the idea of exploration of the CGLE dynamics as a laser model, where the use of both quintic terms is essential. Hence, it is likely that the existence of DSRs will be unveiled in an increasing number of theoretical and experimental situations as well.

\section{ACKNOWLEDGMENTS}

J. M. Soto-Crespo acknowledges support from the Spanish Ministerio de Ciencia e Innovación under contracts FIS2006-03376 and FIS2009-09895. A. Ankiewicz and N. Akhmediev acknowledge the support of the Australian Research Council (ARC) (Discovery Project DP0985394).

\section{REFERENCES}

1. V. L. Kalashnikov, E. Podivilov, A. Chernykh, S. Naumov, A. Fernandez, R. Graf, and A. Apolonski, "Approaching the microjoule frontier with femtosecond laser oscillators: theory and comparison with experiment," New J. Phys. 7, 217 (2005).

2. T. Schreiber, C. Nielsen, B. Ortac, J. Limpert, and A. Tunnermann, "Microjoule-level all-polarization-maintaining femtosecond fiber source," Opt. Lett. 31, 574-576 (2006).

3. A. Chong, W. Renninger, and F. Wise, "All-normaldispersion femtosecond fiber laser with pulse energy above 20 nJ," Opt. Lett. 32, 2408-2410 (2007).

4. S. Kobtsev, S. Kukarin, and Y. Fedotov, "Ultra-low repetition rate mode-locked fiber laser with high-energy pulses," Opt. Express 16, 21936-21941 (2008).

5. C. Lecaplain, B. Ortaç, and A. Hideur, "High-energy femtosecond pulses from a dissipative soliton fiber laser," Opt. Lett. 34, 3731-3733 (2009).

6. S. Lefrançois, K. Kieu, Y. Deng, J. Kafka, and F. Wise, "Scaling of dissipative soliton fiber lasers to megawatt peak powers by use of large-area photonic crystal fiber," Opt. Lett. 35, 1569-1571 (2010).

7. E. Snitzer, H. Po, F. Hakimi, R. Tumminelli, and B. McCollum, "Double-clad, offset-core Nd fiber laser" in Optical Fiber Sensors, Vol. 2 of 1988 OSA Technical Digest Series (Optical Society of America, 1988), paper PD5.

8. B. Ortaç, J. Limpert, and A. Tunnermann, "High-energy femtosecond $\mathrm{Yb}$-doped fiber laser operating in the anomalous dispersion regime," Opt. Lett. 32, 2149-2151 (2007).

9. A. Fernandez, T. Fuji, A. Poppe, A. Fürbach, F. Krausz, and A. Apolonski, "Chirped-pulse oscillators: a route to highpower femtosecond pulses without external amplification," Opt. Lett. 29, 1366-1368 (2004)

10. N. Akhmediev, J. M. Soto-Crespo, and Ph. Grelu, "Roadmap to ultra-short record high-energy pulses out of laser oscillators," Phys. Lett. A 372, 3124-3128 (2008).

11. K. Tamura, E. P. Ippen, and H. A. Haus, "Pulse dynamics in stretched-pulse fiber lasers," Appl. Phys. Lett. 67, 158-160 (1995).

12. D. Anderson, M. Desaix, M. Karlsson, M. Lisak, and M. Quiroga-Teixeiro, "Wave-breaking-free pulses in nonlinear optical fibers," J. Opt. Soc. Am. B 10, 1185-1190 (1993).

13. F. Ilday, J. Buckley, W. Clark, and F. Wise, "Self-similar evolution of parabolic pulses in a laser," Phys. Rev. Lett. 92, 213902 (2004).

14. D. Strickland and G. Mourou, "Compression of amplified chirped optical pulses," Opt. Commun. 56, 219-221 (1985).

15. V. L. Kalashnikov and A. Apolonski, "Chirped-pulse oscillators: A unified standpoint,” Phys. Rev. A 79, 043829 (2009).

16. I. Aranson and L. Kramer, "The world of the complex Ginzburg-Landau equation," Rev. Mod. Phys. 74, 99-143 (2002).

17. N. Akhmediev and A. Ankiewicz, eds., Dissipative Solitons (Springer, 2005).

18. J. M. Soto-Crespo, M. Grapinet, Ph. Grelu, and N. Akhmediev, "Bifurcations and multiple-period soliton pulsations in a passively mode-locked fiber laser," Phys. Rev. E 70, $066612(2004)$.

19. S. Cundiff, J. M. Soto-Crespo, and N. Akhmediev, "Experimental evidence for soliton explosions," Phys. Rev. Lett. 88, 073903 (2002).

20. Ph. Grelu, F. Belhache, F. Gutty, and J. M. Soto-Crespo, "Relative phase locking of pulses in a passively mode-locked fiber laser," J. Opt. Soc. Am. B 20, 863-870 (2003).

21. N. Akhmediev, J. M. Soto-Crespo, M. Grapinet, and Ph. Grelu, "Dissipative soliton interactions inside a fiber laser cavity," Opt. Fiber Technol. 11, 209-228 (2005).

22. W. Chang, A. Ankiewicz, J. M. Soto-Crespo, and N. Akhmediev, "Dissipative soliton resonances," Phys. Rev. A 78, 023830 (2008).

23. W. Chang, J. M. Soto-Crespo, A. Ankiewicz, and N. Akhmediev, "Dissipative soliton resonances in the anomalous dispersion regime," Phys. Rev. A 79, 033840 (2009).

24. X. Liu, "Pulse evolution without wave breaking in a strongly dissipative-dispersive laser system," Phys. Rev. A 81, 053819 (2010).

25. S. Chen, "Theory of dissipative solitons in complex Ginzburg-Landau systems,” Phys. Rev. E 78, 025601(R) (2008).

26. V. L. Kalashnikov, "Chirped dissipative solitons of the complex cubic-quintic nonlinear Ginzburg-Landau equation," Phys. Rev. E 80, 046606 (2009); "Chirped dissipative solitons," arXiv:1001.4918.

27. W. Chang, A. Ankiewicz, J. M. Soto-Crespo, and N. Akhmediev, "Dissipative soliton resonances in laser models with parameter management," J. Opt. Soc. Am. B 25, 1972-1977 (2008).

28. A. Komarov, H. Leblond, and F. Sanchez, "Quintic complex Ginzburg-Landau model for ring fiber lasers," Phys. Rev. E 72, 025604(R) (2005)

29. E. Ding and N. Kutz, "Operating regimes, split-step modeling, and the Haus master mode-locking model," J. Opt. Soc. Am. B 26, 2290-2300 (2009). 